\title{
Ablación de taquicardia supraventricular resistente a terapia médica y a cardioversión eléctrica en una gestante
}

\author{
Edison Muñoz Ortiz ${ }^{\mathrm{a}, \mathrm{b}, *}$, Juan Fernando Agudelo ${ }^{\mathrm{c}}$, Jesús Velásquez ${ }^{\mathrm{d}, \mathrm{e}}$, \\ Edwin Arévalo ${ }^{\mathrm{a}, \mathrm{b}}$ y Jairo Gándara Ricardo ${ }^{\mathrm{a}}$
}

a Clínica de embarazo y enfermedad cardiac, Departamento Cardiovascular, Hospital Universitario San Vicente Fundación, Medellín, Colombia

b Sección de Cardiología, Departamento de Medicina Interna, Universidad de Antioquia, Medellín, Colombia

c Departamento Cardiovascular, Hospital Universitario San Vicente Fundación, Medellín, Colombia

d Clínica de embarazo y enfermedad cardiac, Departamento de Gineco-Obstetricia, Hospital Universitario San Vicente Fundación, Medellín, Colombia

e Sección de Perinatología y alto riesgo obstétrico, Departamento de Obstetricia y Ginecología, Universidad de Antioquia, Medellín, Colombia

Recibido el 3 de septiembre de 2016; aceptado el 3 de abril de 2017

Disponible en Internet el 8 de julio de 2017

\section{PALABRAS CLAVE \\ Embarazo; \\ Arritmia; \\ Taquicardia \\ supraventricular; \\ Ablación}

\begin{abstract}
Resumen Las arritmias cardiacas son complicaciones frecuentes durante el embarazo, y de hecho se ha considerado que este estado incrementa la incidencia de arritmias en pacientes con y sin enfermedad cardiaca estructural. El manejo de las arritmias en la paciente embarazada debe tener en cuenta no solo el bienestar materno sino el fetal, característica que restringe muchas de las terapias usadas en población no embarazada; sin embargo, es poco frecuente la falta de respuesta a terapia médica y cardioversión eléctrica. Se presenta el caso de una paciente gestante con taquicardia supraventricular por reentrada por vía accesoria sin respuesta a diferentes medidas farmacológicas y a varios intentos de cardioversión eléctrica, que requirió estudio electrofisiológico y ablación emergentes. Adicionalmente, se listan algunos aspectos claves sobre el tema.

(C) 2017 Sociedad Colombiana de Cardiología y Cirugía Cardiovascular. Publicado por Elsevier España, S.L.U. Este es un artículo Open Access bajo la licencia CC BY-NC-ND (http:// creativecommons.org/licenses/by-nc-nd/4.0/).
\end{abstract}

\footnotetext{
* Autor para correspondencia.

Correo electrónico: edisonhoy@yahoo.es (E. Muñoz Ortiz).
} 


\section{KEYWORDS}

Pregnancy;

Arrhythmia;

Supraventricular

tachycardia;

Ablation

\section{Ablation of supraventricular tachycardia resistant to medical treatment and electrical cardioversion in a pregnant woman}

Abstract Cardiac arrhythmias are common complications during pregnancy, and it appears that the incidence of arrhythmias has been increasing in patients with and without structural cardiac disease. The management of arrhythmias in the pregnant patient must not only take into account the well-being of the mother, but also that of the foetus, a fact that restricts many of the therapies used in the non-pregnant population. However, the lack of response to medical treatment and electrical cardioversion is rare.

The case is presented of a pregnant patient with supraventricular re-entry tachycardia with no response to different pharmacological measures and to several attempts of electrocardioversion that required an electrophysiological work-up and resulting ablation. Some key aspects on the subject are also mentioned.

(c) 2017 Sociedad Colombiana de Cardiología y Cirugía Cardiovascular. Published by Elsevier España, S.L.U. This is an open access article under the CC BY-NC-ND license (http:// creativecommons.org/licenses/by-nc-nd/4.0/).

\section{Introducción}

Las arritmias cardiacas son complicaciones frecuentes durante el embarazo, y de hecho se ha considerado que el embarazo incrementa la incidencia de arritmias en pacientes con y sin enfermedad cardiaca estructural ${ }^{1-4}$. Las arritmias supraventriculares son las que más frecuentemente se presentan, ocurriendo de forma sostenida hasta en $1,3 \%$ de las mujeres embarazadas sin enfermedad cardiaca estructural, de ellas, el 34\% tienen el primer episodio de la arritmia durante el embarazo y 29 a $44 \%$ tienen una exacerbación de arritmias ya conocidas ${ }^{5}$.

El tratamiento agudo de la arritmia suele hacerse con medicamentos como adenosina, metoprolol o la realización de cardioversión eléctrica, debido a que se consideran seguros para el feto, y generalmente logran abortar el evento arrítmico; mientras que la ablación por radiofrecuencia, que es un procedimiento curativo de la arritmia en muchos casos, es raramente realizado durante el embarazo pues la radiación debida a la exposición a rayos $X$ puede ser también dañina para el feto; sin embargo, en pacientes en quienes los medicamentos no han sido efectivos en quitar una arritmia aguda, o prevenir recurrencias, el procedimiento de ablación puede ser la única alternativa terapéutica ${ }^{5}$.

Se describe a continuación un caso de una gestante con taquicardia supraventricular paroxística por fenómeno de reentrada por vía accesoria, refractaria a terapia médica y a cardioversión eléctrica, lo que llevó a la realización de un procedimiento de mapeo y ablación urgente con un buen desenlace materno-fetal.

\section{Caso}

Paciente de 29 años, multigestante (5 embarazos previos), sin antecedentes de importancia, quien ingresó al servicio de urgencias obstétricas del Hospital Universitario de San Vicente Fundación en horas de la madrugada, remitida de un hospital de primer nivel de complejidad, con embarazo de $26+1$ semanas, cuadro clínico de 12 horas conformado por palpitaciones, dolor en región precordial irradiado a cuello, disnea y necesidad de suplemento de oxígeno para saturar adecuadamente. Refería que desde hacía siete meses presentaba episodios de palpitaciones que duraban 10 a 15 minutos y se resolvían de manera espontánea por lo que no había consultado. Adicionalmente, relataba síntomas irritativos urinarios de unos dos días de evolución. Al ingreso en horas de la noche fue evaluada inicialmente por el servicio de Gineco-Obstetricia y Medicina interna, encontrando presión arterial de $80 / 40 \mathrm{~mm} \mathrm{Hg}$, frecuencia cardiaca de 180 latidos por minuto, frecuencia respiratoria de 24 respiraciones por minuto, saturación de oxígeno de $94 \%$, no soplos ni punto de máximo impulso desplazado, y auscultación pulmonar normal. No edemas, y perfusión distal y estado de consciencia normales. La altura uterina era 24 centímetros, no había actividad uterina y la fetocardia fue de 156 latidos por minuto. Se tomó electrocardiograma (fig. 1), el cual se interpretó como taquicardia supraventricular paroxística; adicionalmente se sospechó sepsis de origen urinario. Se inició adenosina $6 \mathrm{mg}$ IV, y ante la falta de respuesta se suministraron 2 dosis adicionales de $12 \mathrm{mg}$ cada una, sin lograr terminación de la arritmia. Debido a que las cifras tensionales permanecían limítrofes, se decidió realizar cardioversión eléctrica con equipo bifásico con $50 \mathrm{~J}$ sin respuesta, más otras dos cardioversiones, una con $100 \mathrm{~J}$ y otra con $200 \mathrm{~J}$; no obstante, en ninguna de las cardioversiones se logró pasar a ritmo sinusal. Persistía con frecuencia cardiaca alrededor de 180 latidos por minuto. Ante la falta de respuesta y no evidencia de deterioro, se le aplicaron $15 \mathrm{mg}$ de metoprolol venoso en dosis tituladas, logrando por momentos disminución de la frecuencia cardiaca hasta 160 latidos por minuto, pero sin convertir a ritmo sinusal, y retornando rápidamente a frecuencias cercanas a los 180 latidos por minuto. Se decidió iniciar esquema de maduración pulmonar fetal con betametasona.

Los paraclínicos no mostraron alteraciones electrolíticas, y la función renal y las pruebas tiroideas fueron normales. El hemograma evidenció 15.700 leucocitos y hemoglobina de $9,9 \mathrm{~g} / \mathrm{dl}$. Fue evaluada por Cardiología clínica cuando completaba 20 horas de evolución de las palpitaciones, y al leer el electrocardiograma se consideró posible taquicardia supraventricular paroxística por reentrada por vía 


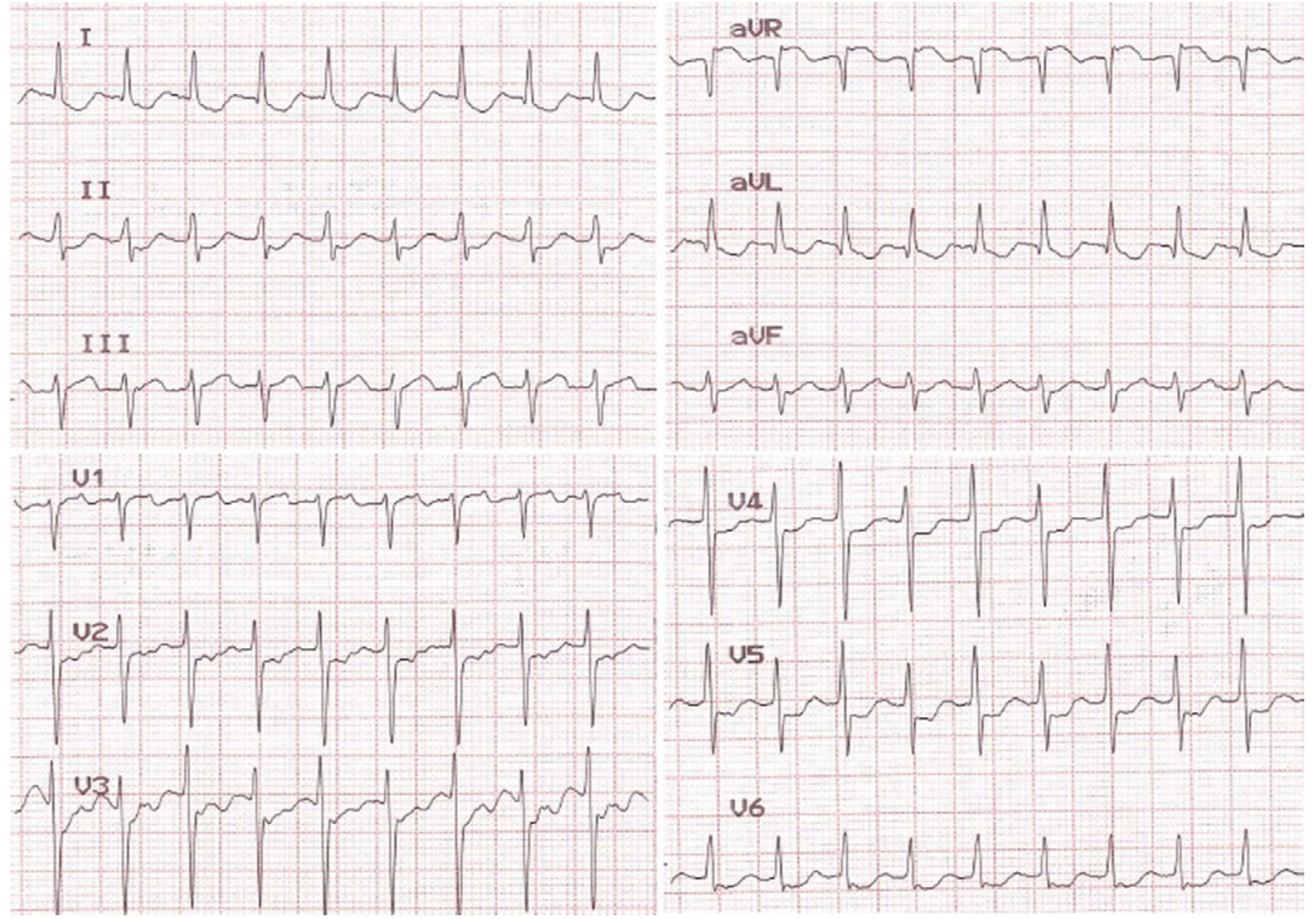

Figura 1 Electrocardiograma de ingreso en el que se evidencia taquicardia de complejos estrechos, rítmica, RP mayor de $90 \mathrm{~ms}$, además de alternancia eléctrica, por tanto se consideró taquicardia supraventricular por reentrada aurículo-ventricular (vía accesoria).
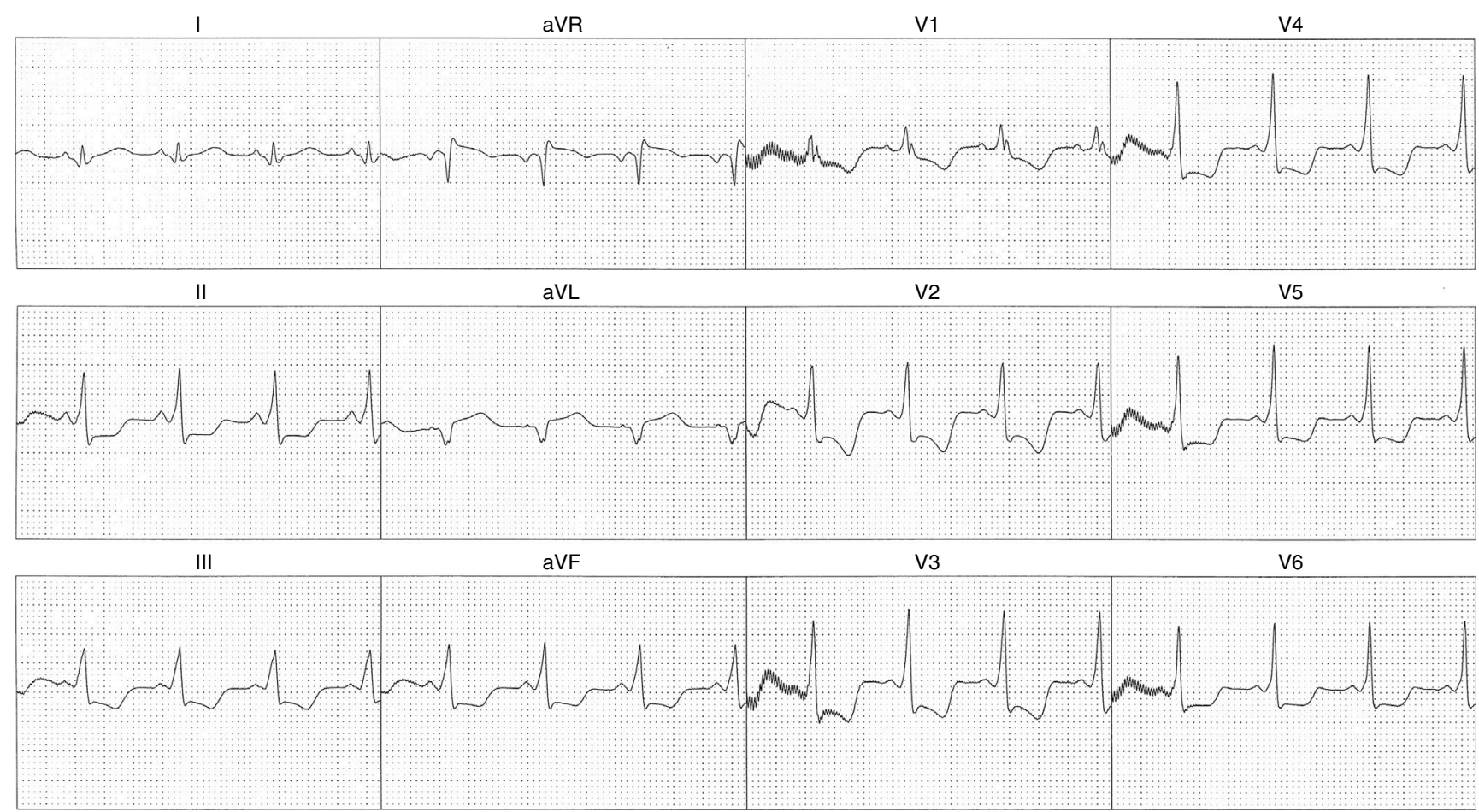

Figura 2 Retorno a ritmo sinusal luego de estimulación con catéter durante estudio electrofisiológico. Se evidencia ritmo sinusal, con PR corto y QRS ancho por presencia de onda delta, configurándose un síndrome de Wolff-Parkinson-White debido a un evento arrítmico previo. 


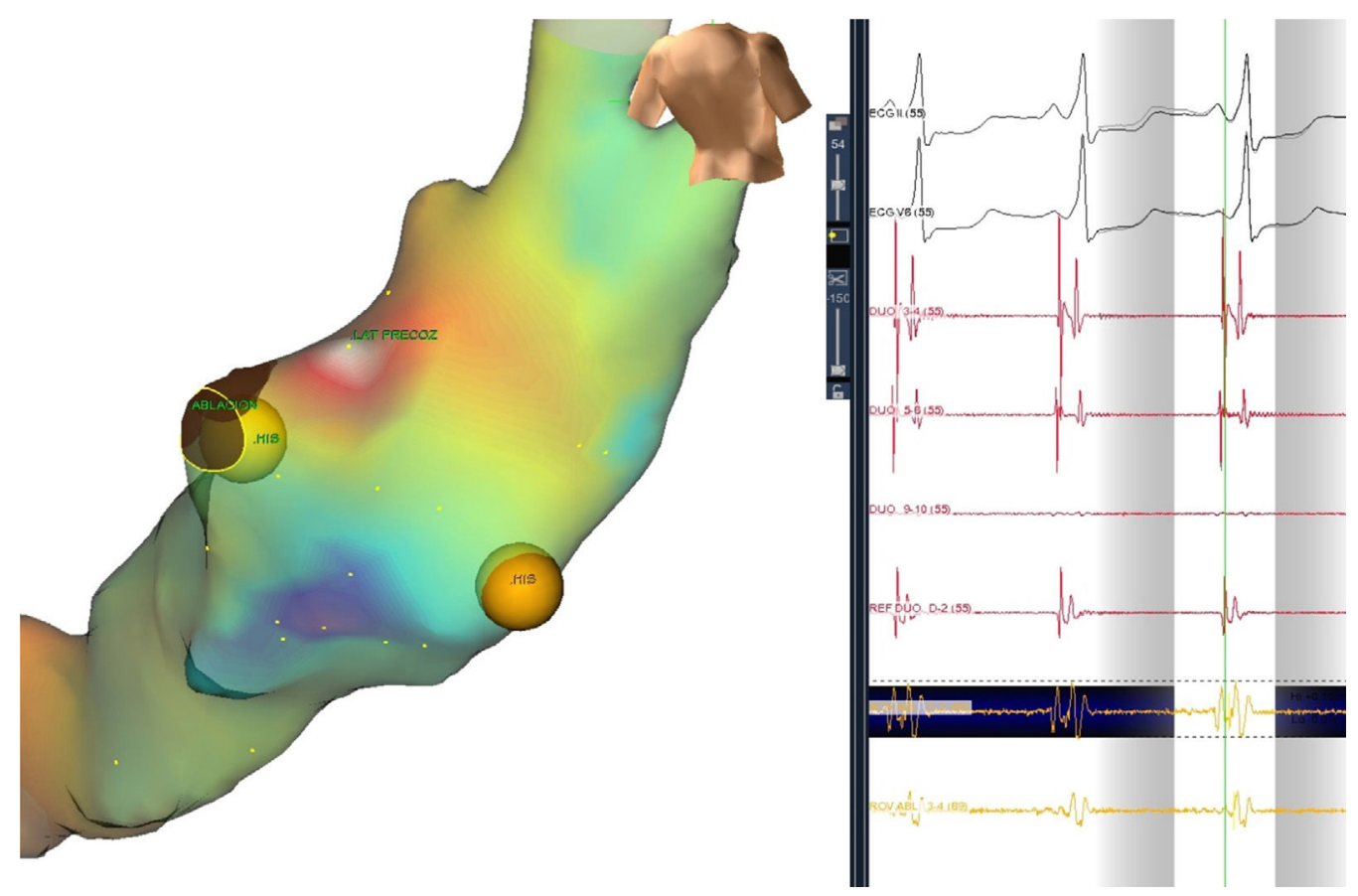

Figura 3 Mapa de activación del anillo mitral trazado con sistema de mapeo tridimensional Ensite de St. Jude Medical. Se aprecia precocidad en la región lateral de dicho anillo con fusión de electrograma.

accesoria, sin respuesta a terapia médica ni a cardioversión eléctrica. Se hicieron dos intentos adicionales con equipo bifásico de $200 \mathrm{~J}$ ubicando las paletas en forma anteroposterior; sin embargo estos dos intentos adicionales de retornar a ritmo sinusal fueron nuevamente fallidos. En ese momento no presentaba deterioro de las cifras de presión arterial $(92 / 58 \mathrm{~mm} \mathrm{Hg})$, y su frecuencia cardiaca era de 184 latidos por minuto. Ante la falta de respuesta a las diferentes medidas, se solicitó evaluación por Electrofisiología y se decidió llevar a procedimiento de estudio electrofisiológico, mapeo y ablación emergente.

Se inició estudio electrofisiológico con protección abdominal plomado, en el que se apreció taquicardia regular con QRS estrecho con longitud de ciclo de $333 \mathrm{~ms}$ con intervalo ventrículo-auricular (VA) largo, compatible con taquicardia por movimiento circular ortodrómico. Se intentó maniobra de encarrilamiento desde el ventrículo, con lo que se terminó la taquicardia. Se tomó electrocardiograma donde se evidenció presencia de PR corto con onda delta compatible con síndrome de Wolff-Parkinson-White (fig. 2). Se apreció conducción aurículo-ventricular excéntrica con mayor precocidad en relación con los electrodos distales ( 1 y 2 ) con lo que se confirmó una localización lateral izquierda de la vía accesoria. Con sistema de mapeo tridimensional ENSITE de ST Jude (Mineapolis, EUA), se mapeó la región lateral del anillo mitral hasta documentar fusión de los electrogramas con precocidad de hasta $30 \mathrm{~ms}$. En dicho sitio se aplicaron lesiones con energía de radiofrecuencia bajo control de temperatura (40 Watts) con lo que se logró la desaparición de la preexcitación a los tres segundos. Se documentó posteriormente disociación VA. A continuación, se completó protocolo de estimulación atrial y ventricular con dos longitudes de ciclo y hasta tres extraestímulos sin lograr documentar arritmia alguna. Al final del procedimiento se documentó permanencia de la disociación VA, considerándose un procedimiento exitoso (fig. 3). El tiempo de radiación fue de 25 minutos. Durante el procedimiento se tuvo además vigilancia de la frecuencia cardiaca fetal, la cual estuvo todo el tiempo en límites normales.

El urocultivo y los hemocultivos fueron negativos. Durante la estancia hospitalaria no presentó recurrencia de síntomas y se dio de alta a las 48 horas de procedimiento.

Continuó su embarazo sin complicaciones hasta la semana $40+3$, cuando ingresó en fase activa del trabajo de parto, teniendo parto vértice espontáneo sin complicaciones con producto de género masculino, también sin complicaciones.

\section{Discusión}

Las arritmias suelen ser relativamente frecuentes y exacerbarse durante el embarazo. El riesgo de presentar taquicardia supraventricular (TSV) en una embarazada respecto a la población no gestante se ha calculado en $5,1^{6}$. Sin embargo la mayoría de las arritmias en el embarazo son benignas.

La explicación fisiopatológica de porqué las arritmias aumentan durante el embarazo no es clara, pero se considera que son producto de la interacción de cambios hemodinámicos, hormonales y autonómicos propios de la gestación. Se destacan el aumento del volumen sanguíneo efectivo circulante de 30 a 50\% a partir de las 8 semanas de gestación, con su mayor efecto alrededor de las 34 semanas; además, el aumento del gasto cardiaco secundario al incremento en el volumen latido del $35 \%$ y el incremento de la frecuencia cardiaca en un $15 \%{ }^{7}$. Por otro lado, el aumento del volumen plasmático produce estiramiento de 
Tabla 1 Recomendaciones para uso de antiarrítmicos en el embarazo ${ }^{10}$

\begin{tabular}{|c|c|c|c|c|c|c|}
\hline Medicamento & $\begin{array}{l}\text { Clase } \\
\text { Vaugham } \\
\text { Williams }\end{array}$ & $\begin{array}{l}\text { Categoría } \\
\text { FDA }\end{array}$ & $\begin{array}{l}\text { Efectos adversos } \\
\text { potenciales }\end{array}$ & $\begin{array}{l}\text { Indicación primaria } \\
\text { en la madre }\end{array}$ & $\begin{array}{l}\text { Uso durante } \\
\text { la lactancia }\end{array}$ & Observaciones \\
\hline Lidocaína & IB & B & $\begin{array}{l}\text { Efectos adversos en } \\
\text { el SNC, bradicardia }\end{array}$ & $\begin{array}{l}\text { TV, arritmias debidas } \\
\text { a toxicidad por } \\
\text { digoxina }\end{array}$ & $\begin{array}{l}\text { Puede } \\
\text { usarse }\end{array}$ & $\begin{array}{l}\text { Larga historia } \\
\text { de seguridad. } \\
\text { Evitar en } \\
\text { sufrimiento } \\
\text { fetal }\end{array}$ \\
\hline Propafenona & IC & $\mathrm{C}$ & $\begin{array}{l}\text { Incremento en } \\
\text { mortalidad en } \\
\text { pacientes con previo } \\
\text { IAM. Leve efecto } \\
\text { betabloqueador }\end{array}$ & TSV y TV & Desconocido & $\begin{array}{l}\text { Pocos datos } \\
\text { disponibles }\end{array}$ \\
\hline $\begin{array}{l}\text { Beta- } \\
\text { bloqueadores }\end{array}$ & ॥ & $C / D$ & $\begin{array}{l}\text { Retardo del } \\
\text { crecimiento } \\
\text { intrauterino, } \\
\text { bradicardia fetal, } \\
\text { hipoglicemia, apnea } \\
\text { fetal }\end{array}$ & $\begin{array}{l}\text { TSV, TV idiopática, } \\
\text { control de respuesta } \\
\text { ventricular en FA }\end{array}$ & $\begin{array}{l}\text { Evitar } \\
\text { atenolol. } \\
\text { Metoprolol y } \\
\text { propranolol } \\
\text { pueden } \\
\text { usarse }\end{array}$ & $\begin{array}{l}\text { Generalmente } \\
\text { bien tolerados; } \\
\text { evitar durante } \\
\text { el primer } \\
\text { trimestre, } \\
\text { preferir los } \\
\text { cardioselectivos }\end{array}$ \\
\hline Amiodarona & III & $\mathrm{D}$ & $\begin{array}{l}\text { Hipotiroidismo fetal, } \\
\text { prematuridad, bajo } \\
\text { peso al nacer, } \\
\text { malformaciones } \\
\text { congénitas }\end{array}$ & $\begin{array}{l}\text { Arritmias } \\
\text { ventriculares } \\
\text { amenazantes para la } \\
\text { vida }\end{array}$ & Evitar & $\begin{array}{l}\text { Evitar si es } \\
\text { posible, } \\
\text { especialmente } \\
\text { durante el } \\
\text { primer } \\
\text { trimestre }\end{array}$ \\
\hline Verapamilo & IV & C & $\begin{array}{l}\text { Hipotensión materna, } \\
\text { bloqueo cardiaco y } \\
\text { bradicardia fetal }\end{array}$ & $\begin{array}{l}\text { TSV, TV idiopática, } \\
\text { control de respuesta } \\
\text { ventricular en FA }\end{array}$ & $\begin{array}{l}\text { Puede } \\
\text { usarse }\end{array}$ & $\begin{array}{l}\text { Relativamente } \\
\text { bien tolerado, } \\
\text { pero pueden } \\
\text { preferirse } \\
\text { opciones más } \\
\text { seguras }\end{array}$ \\
\hline Diltiazem & IV & C & $\begin{array}{l}\text { Datos limitados. } \\
\text { Similar a verapamilo }\end{array}$ & $\begin{array}{l}\text { TSV, control de } \\
\text { respuesta ventricular } \\
\text { en FA }\end{array}$ & $\begin{array}{l}\text { Puede } \\
\text { usarse }\end{array}$ & $\begin{array}{l}\text { Se prefiere el } \\
\text { verapamilo por } \\
\text { mayor } \\
\text { experiencia }\end{array}$ \\
\hline Adenosina & No aplica & C & $\begin{array}{l}\text { Disnea y bradicardia } \\
\text { materna }\end{array}$ & $\begin{array}{l}\text { Terminación aguda de } \\
\text { TSV }\end{array}$ & $\begin{array}{l}\text { Desconocido } \\
\text { pero proba- } \\
\text { blemente } \\
\text { bien } \\
\text { tolerada }\end{array}$ & $\begin{array}{l}\text { Primera opción } \\
\text { para } \\
\text { terminación de } \\
\text { TSV que } \\
\text { dependan del } \\
\text { nodo AV }\end{array}$ \\
\hline Digoxina & No aplica & $\mathrm{C}$ & Bajo peso al nacer & $\begin{array}{l}\text { TSV, control de } \\
\text { respuesta ventricular } \\
\text { en FA }\end{array}$ & $\begin{array}{l}\text { Puede } \\
\text { usarse }\end{array}$ & $\begin{array}{l}\text { Gran } \\
\text { experiencia en } \\
\text { su uso }\end{array}$ \\
\hline
\end{tabular}

FA: fibrilación auricular; IAM: infarto agudo de miocardio; SNC: sistema nervioso central;

TSV: taquicardia supraventricular; TV: taquicardia ventricular.

los miocitos auriculares y ventriculares, que podría causar posdespolarizaciones tempranas, conducción lenta, acortamiento del periodo refractario y dispersión espacial a través de canales iónicos activados por el estiramiento ${ }^{7,8}$. La dilatación de las fibras miocárdicas y el aumento del tamaño cardiaco, podrían hacer más fáciles de sostener los circuitos de reentrada al aumentar la longitud del trayecto implicado en el fenómeno de reentrada ${ }^{7}$. En cuanto a los cambios hormonales y autonómicos, el estradiol y la progesterona pueden aumentar la aparición de arritmias. Los estrógenos pueden incrementar los receptores adrenérgicos ${ }^{7}$.

Cuando se produce una arritmia hemodinamicamente significativa durante el embarazo, el manejo médico se ve limitado por varios factores, entre ellos la gravedad de la arritmia y las consecuencias de la inestabilidad hemodinámica tanto para la madre como para el feto pues la presión arterial durante la arritmia es más baja que lo habitual, hecho que puede reducir la perfusión placentaria ${ }^{10}$. 
Adicionalmente, se limita el uso de medicamentos antiarrítmicos tradicionales por sus efectos sobre el feto (prematurez, restricción del crecimiento intrauterino, enfermedades cardiacas congénitas) ${ }^{7}$.

No todas las arritmias durante el embarazo se pueden manejar de forma conservadora, ya que ante taquicardia, existe riesgo de desarrollar taquicardiomiopatía más del $30 \%$ del tiempo 9 .

En la tabla 1 se presentan los medicamentos antiarrítmicos utilizados en nuestro medio, su clasificación de seguridad en el embarazo según la FDA y los efectos sobre el feto. En términos generales, los medicamentos antiarrítmicos se consideran categoría C (hay posibilidad de daño fetal si se administra durante el embarazo, pero puede usarse si el beneficio supera los riesgos) y $D$ (evidencia de riesgo. Estudios en humanos han mostrado evidencia de daño fetal. Sin embargo, podría usarse si el potencial beneficio de su uso en la mujer embarazada sobrepasa el riesgo) por la FDA ${ }^{10}$.

Por otro lado, la cardioversión eléctrica es segura en todas las etapas del embarazo, aunque con un riesgo teórico de inicio de parto pretérmino en etapas avanzadas del embarazo. Esta intervención se considera la terapia de elección en las pacientes con inestabilidad hemodinámica, si bien hay reportes de casos de necesidad de cesárea urgente por arritmias fetales después de una cardioversión, de ahí que sea necesaria la monitorización del feto ${ }^{7}$.

La ablación con catéter, que sería una opción de terapia curativa, se reserva solo para gestantes con arritmias severas sin respuesta a la terapia médica. Se tienen pocos estudios en las pacientes embarazadas y suele no usarse debido al riesgo para el feto de la radiación empleada en la fluoroscopia; entre estos se incluyen, al parecer, mayor riesgo de padecer cáncer en el futuro ${ }^{11}$. Tradicionalmente, la fluoroscopia se ha utilizado para la visualización de los catéteres durante el procedimiento de ablación; en casos reportados los tiempos de exposición a la radiación van de 0 segundos a 36 minutos $^{5}$. Sin embargo esta puede minimizarse utilizando protección plomada abdominal, tiempos de exposición cortos y, más recientemente, sistemas electroanatómicos $3 \mathrm{D}$ que minimicen aún más el uso de radiación ${ }^{5}$.

\section{Conclusiones}

El cuidado de las gestantes con arritmias cardiacas debe estar a cargo de un equipo multidisciplinario conformado por gineco-obstetras, cardiólogos y electrofisiólogos expertos y familiarizados con el impacto de la gestación en la función cardiovascular. El uso adecuado de los medicamentos, la cardioversión y ablación si es del caso, con medidas de protección, y las nuevas herramientas en electrofisiología deben ser las de elección en los casos de arritmias refractarias al manejo médico.

\section{Responsabilidades éticas}

Protección de personas y animales. Los autores declaran que para esta investigación no se han realizado experimentos en seres humanos ni en animales.
Confidencialidad de los datos. Los autores declaran que han seguido los protocolos de su centro de trabajo sobre la publicación de datos de pacientes.

Derecho a la privacidad y consentimiento informado. Los autores declaran que en este artículo no aparecen datos de pacientes.

\section{Financiación}

Ninguna.

\section{Conflicto de intereses}

Ninguno.

\section{Bibliografía}

1. Page RL, Joglar JA, Caldwell MA, Calkins H, Conti JB, Deal BJ, et al. 2015 ACC/AHA/HRS Guideline for the Management of Adult Patients With Supraventricular Tachycardia: A Report of the American College of Cardiology/American Heart Association Task Force on Clinical Practice Guidelines and the Heart Rhythm Society. J Am Coll Cardiol. 2016;67:e27-115.

2. Siu SC, Sermer M, Colman JM, Alvarez AN, Mercier LA, Morton $B C$, et al. Prospective multicenter study of pregnancy outcomes in women with heart disease. Circulation. 2001;104: 515-21.

3. Silversides CK, Harris L, Haberer K, Sermer M, Colman JM, Siu SC. Recurrence rates of arrhythmias during pregnancy in women with previous tachyarrhythmia and impact on fetal and neonatal outcomes. Am J Cardiol. 2006;97:1206-12.

4. Shotan A, Ostrzega E, Mehra A, Johnson JV, Elkayam U. Incidence of arrhythmias in normal pregnancy and relation to palpitations, dizziness, and syncope. Am J Cardiol. 1997;79:1061-4.

5. Szumowski L, Szufladowicz E, Orczykowski M, Bodalski R, Derejko P, Przybylski A, et al. Ablation of severe drug-resistant tachyarrhythmia during pregnancy. J Cardiovasc Electrophysiol. 2010;21:877-82.

6. Tawam M, Levine J, Mendelson M, Goldberger J, Dyer A, Kadish A. Effect of pregnancy on paroxysmal supraventricular tachycardia. Am J Cardiol. 1993;72:838-40.

7. Enriquez AD, Economy KE, Tedrow UB. Contemporary management of arrhythmias during pregnancy. Circ Arrhythm Electrophysiol. 2014;7:961-7.

8. Ninio DM, Saint DA. The role of stretch-activated channels in atrial fibrillation and the impact of intracellular acidosis. Prog Biophys Mol Biol. 2008;97(2-3):401-16.

9. Bigelow AM, Crane SS, Khoury FR, Clark JM. Catheter ablation of supraventricular tachycardia without fluoroscopy during pregnancy. Obstet Gynecol. 2015;125:1338-41.

10. Joglar JA, Page RL. Management of arrhythmia syndromes during pregnancy. Curr Opin Cardiol. 2014;29:36-44.

11. Wakeford R, Little MP. Risk coefficients for childhood cancer after intrauterine irradiation: a review. Int J Radiat Biol. 2003;79:293-309. 